\title{
Astrochemistry: From Molecular Clouds to Planetary Systems; Conference Summary
}

\author{
D. A. Williams \\ Department of Physics and Astronomy, University College London, \\ Gower Street, London WC1E 6BT, UK
}

\begin{abstract}
Some thoughts on the state of our subject, as described in the presentations at the Symposium, are given here.
\end{abstract}

\section{Introduction}

What a feast of science has been presented at this Symposium! New observations have delighted us, new theories have amazed us, and new problems have intrigued us. We are fortunate to be living at such a time and able to take part in what one might call the 'big bang' of molecular astrophysics. There has never been in our subject a time quite like this for revelation, confusion, and excitement. We know that our subject has great breadth, and this Symposium has shown that in terms of its depth, it is now advancing very rapidly. In fact, the activity in our subject is so great that, for the first time in this series of Symposia, the range of science discussed had to be curtailed.

In his opening address, Dalgarno reminded us of the breadth of our subject. It now contributes in a fundamental way to a huge swathe of astronomy from the early Universe to the formation of stars and planets and the origin of life. In some of these areas, astrochemistry is astronomy; i.e. it represents the only effective tool we have. Some of the astrochemical contributions are well established and mature, others are more speculative. We are lucky to have the opportunity of contributing to several of these areas of astronomy, for the essential approach is the same: how do the molecular processes operating at the microscopic level determine the gross properties of the system at the macroscopic level? Insights gained in one area do help us to understand others, too, and sometimes it appears that the rewards of insight gained far outweigh the efforts made.

The Symposium has reminded us of the depth of our subject, i.e. its ability to give us insight. Dalgarno called it 'a blend of astronomy and chemistry, of extraordinary power' and remarked that 'with ISO, astrochemistry had come of age.' Astrochemistry is no longer merely interesting, but is truly useful. Nevertheless, the subject is not so well established that it can no longer surprise or shock us. Results given at the Symposium have demonstrated both aspects: ISO results have contributed hugely to many aspects of our understanding of interstellar clouds and circumstellar matter, but what in Heaven can be the source of the excitation of $\mathrm{H}_{2} J=27$ in Orion Peak 1, as reported by Bertoldi? This result seems revolutionary! Again, while ISO gives us much information about the solid phase of oxygen-bearing molecules, as van Dishoeck and Ehrenfreund 
reminded us, the preliminary SWAS results described by Melnick have been a shock to those who, like me, have complacently assumed that SWAS would simply confirm our prejudices. The discussions during the Symposium of intriguing results like these questioned the physical basis of the models against which these new results are being assessed. I think that such emphasis on the fallibility of our models is fully justified. Although our chemical descriptions are certainly not complete, it is probable that we have mostly given them more attention than the physical models in which they are embedded. Perhaps this focus on physics will be the thrust of the work before the next Symposium. In fact, the quality of the observational work presented at this Symposium has been so impressive that it is clear that the subject will continue to be observationally led. The models we use are in some cases simply incapable of generating the complexities found in the data.

The pace of advance in depth causes its own problems. As the battle front rolls forward, problems in the rear are ignored, yet may cause insurrections at a later time and put further progress in jeopardy. For example, consider the case of diffuse and translucent clouds. Turner emphasized that these are important test beds for astrochemistry, yet the results that he has obtained, and those of Liszt \& Lucas identifying diffuse cloud polyatomics, call for a review of our understanding, as he pointed out. The pathological case of $\mathrm{CH}^{+}$remains, and our understanding seems not to have advanced since the last Symposium. The detection of $\mathrm{H}_{3}^{+}$by Geballe and collaborators was greeted with relief by all astrochemists, but presents a serious problem of interpretation in diffuse clouds. The models we use for diffuse clouds evidently need revision. Beware, lest rebellions in otherwise quiescent territory put the whole campaign at risk!

I propose in this article to make a few remarks about the various scientific areas included in the Symposium, as represented by the work presented. These are purely personal perceptions about present and future activities in our subject. Then, in Section 3, I shall make some conclusions.

\section{Scientific Areas}

\subsection{Chemistry in pre-stellar cores and low-mass star-forming re- gions}

This is one of the most active of all areas of astrochemistry. Practitioners regard it as one of the main drivers of our subject, and perhaps with good reason; for almost all the aspects of astrochemistry seem to find expression here. It may be because of that high complexity that we are not yet near the stage of a definitive description - at least on the basis of the presentations made at the Symposium. One topic for discussion was the initial state of the cloud and the dynamical model of collapse; and there seemed to be differing views on the importance of these features and whether there would be observational tracers. It is clear, however, that on the microscopic level the gas/dust interaction is important, and that there is a great need for reliable information, a need comparable to that posed by gas phase interstellar chemistry some thirty years ago. One encouraging feature is that this problem may be addressed not only through fundamental studies of surface processes but also through interpretation of the observations. Careful observation of line profiles of several lines of several molecules could give 
some reliable information about effective sticking probabilities. This assumes that proper radiative transfer calculations are performed, a task that Black emphasized was now tractable.

The importance of magnetic fields in low-mass star-formation was established, as was the importance of the chemistry in controlling this cloud support. This significant advance is obscured, however, by the lack of knowledge of large molecules or small grains present as charge carriers in the gas.

However, these potential advances presume that we know how to deal with clumpiness, and how to describe the interaction between simultaneous infall and outflow. Evidently, we are some way from building a comprehensive model, and we must continue to find ways of isolating parts of the problem to study independently. Perhaps, the frankness with which practitioners in this area revealed doubts and weakness should be taken as read for other, less intensively - studied, topics!

\subsection{Chemistry in high-mass star-forming regions}

The impact of ISO observations on our understanding of such regions has been enormous. The ability to observe species in both gas and ice forms is placing useful constraints on models. In general, where comparisons between observations and models were possible, our understanding and insight seemed satisfactory. However, it is difficult to see why the uncertainties and gaps in knowledge in low-mass star-formation do not reappear here. It may be that the modellers have so far been lucky. Herbst remarked: 'Astrochemistry modellers are intrepid, but are they right?' The implied call for caution may be justified. Models used in this area are generally less well-developed than those for low-mass star-forming regions. In particular, the remark by Melinck 'The link between chemistry and dynamics is closer than generally believed' struck a chord with this reviewer. Perhaps the surprising SWAS results will have their resolution in this aspect of the models.

\subsection{Basic molecular processes - gas phase and solid state}

We are in danger of becoming blasé about the beautiful and extensive work on gas phase chemistry carried out over the last thirty years, both in the laboratory and on the computer. In fact, it should still have the power to enthral us, as for example - in the strong appearance of the three-body channel in dissociative recombination of molecular ions, discussed here by Zajfman.

The enormity of the task originally posed by astrochemists, in terms of literally thousands of ion-molecule and neutral-neutral reactions, is difficult to appreciate with hindsight. That work continues, particularly in Rennes, Birmingham, and Taiwan, and without it our subject would be mere speculation. Future projects will include the gas/surface interaction. It is now clearly possible to address these questions in a realistic manner, both in the laboratory and on the computer. Our next Symposium will show great advances in this area.

\subsection{Grains and large molecules}

It is true, as Witt emphasized, that we do not yet have a fully comprehensive model of interstellar dust. Nevertheless, my impression is of a steady advance in understanding of each facet of behaviour of grains, and that combining these 
components of understanding into a comprehensive picture cannot be too far off. One of the particularly interesting developments in this very active area has been the recognition that meteorite inclusions contain unprocessed interstellar grains. Messenger noted the presence of GEMS (glasses with embedded metal and sulphides) in interstellar dust particles; these have been proposed as the 'typical' interstellar silicates. Perhaps by the next Symposium, the characteristics of interstellar dust will be definitively specified, rather than the loose generalities of 'silicates' and 'carbons' that we use now.

The PAH hypothesis has been transformed by a wealth of data from ISO, as Tielens made clear. This represents a great step forward, placing severe constraints on other proposals, and demanding an urgent re-assessment of the role of PAHs in interstellar and circumstellar space.

For example, are they responsible for the diffuse interstellar bands? Certainly, the work of Sarre and his collaborators provides compelling evidence that the DIB carriers - or some of them - are molecules of a size comparable to mid-range PAHs. On the other hand, spectroscopic evidence for linear carbon structures, as described by Maier, is also available. Is it possible that both ring $(\mathrm{PAH})$ and linear structures abound in the interstellar medium? They are present, after all, in the solid phase.

\subsection{Circumstellar disks and the connection to the outer Solar Sys- tem}

This is an area whose beauty and complexity we can as yet only dimly see. The observations of disks and the implications for planet-building are tantalizing, but for definitive observations we must wait for ALMA. There are many questions with unknown answers, as Koerner demonstrated. Chemistry is already contributing significantly, as Dutrey and Aikawa showed, but the physical models have not yet been fully developed. Evidently, they will be complex, as Johnstone argued, and involve several components including PDRs.

The problem of understanding can here be helped by recognising that the Solar System evolved from a circumstellar disk. Astronomical spectroscopy with ISO of disks, laboratory and space experiments, and studies of solar systems are all contributing to progress in this rapidly developing area.

\subsection{Chemistry in the envelopes of late-type stars}

This is one of the great success stories of astrochemistry. Some of the most precise observations in terms of masses, chemistry, and dynamics have provided exquisite tests of models, and a wealth of astronomical information has arisen from this work. This reward seems far in advance of that from other areas of modelling. However, there are 'second order' effects to be explained. These may be related to clumpiness, secular variations, or non-isotropy in the outflow, and there seems to be a need to introduce surface processes into the chemistry of the outflow. Of course, the origin of the dust at the base of the outflow remains a mystery. The transformation of near-isotropic outflows to decidedly bipolar planetary nebulae is, unfortunately, poorly understood. 


\subsection{Outflows, Shocks, PDRs, and Masers}

These phenomena describe, in part, the consequences of the formation of a star on its environment. As such, this is one of the most active areas in astronomy today, and the insight gained into the astronomical events is rather precise. While the origin of outflows remains unclear, the outflows, and particularly the collimated jets at an early stage, constitute a useful probe of star-forming regions. They also are major contributors of energy and momentum, affecting the entire cloud dynamics. The shocks also redistribute material in favour of gas rather than dust, being one of the few phenomena capable of destroying refractory grains. We may anticipate a unified picture of the post-birth environment of stars being available by the next Symposium.

\subsection{Outer solar nebula and planetary atmospheres}

Our understanding of planet formation, as developed for the single example that we knew of (the Solar System), has - not surprisingly - not stood the test of new data, i.e. the detection of massive extra-solar planets. ISO has brought rather detailed information about the composition of the atmospheres of planets. The implication of these new data for the origins of oceans of Earth are severe; and the whole picture of the proto-Solar Nebula and planet formation is under review.

\subsection{The interplanetary medium and comets}

In comets, we have the opportunity to analyse interstellar material at close hand, and in studying meteorites and collected interplanetary dust particles we can isolate individual unprocessed interstellar grains from much processed material. From the perspective of the interstellar chemist, the possibilities opened up by these studies are immense. The implications of work presented at this Symposium are compelling.

From the perspective of interplanetary studies, as was pointed out by Gilmour and Messenger, we need to understand better grain surface chemistry in the interstellar medium before the complete picture embedded in interplanetary material can be made clear.

We have long known of our own interstellar origin, but the science is driving us to a deeper recognition of the importance of the solid phase in modern astronomy.

\section{Conclusions}

Astrochemistry has spread its influence throughout all those parts of astronomy where material is relatively cool $(\$ 4000 \mathrm{~K})$, relatively dense $(\gtrsim 1 \mathrm{H}$ atom $\mathrm{cm}^{-3}$ ), and mostly neutral. Those parameters are met in many astronomical circumstances, particularly in interstellar and circumstellar environments. Star formation occurs in the former, and those newly formed stars interact with the latter. It seems likely that the breadth of our subject is now determined: there may be no new frontiers for astrochemistry, and that at the next Symposium we shall concentrate on the depth. The various areas of our subject are at different stages: diffuse clouds and cool stellar envelopes are well established - though 
here there is still much to do. Newer areas are calling for intense study: the stellar perinatal events; the links to interplanetary matter. Perhaps a good message to take away is the exhortation of Monica Kress: 'The solar nebula should be treated as a process, not an object'; in other words, things interact and evolve but tell a story, too.

The level of excitement in our subject is very high. This meeting has shown us, too, what great fun it all is!

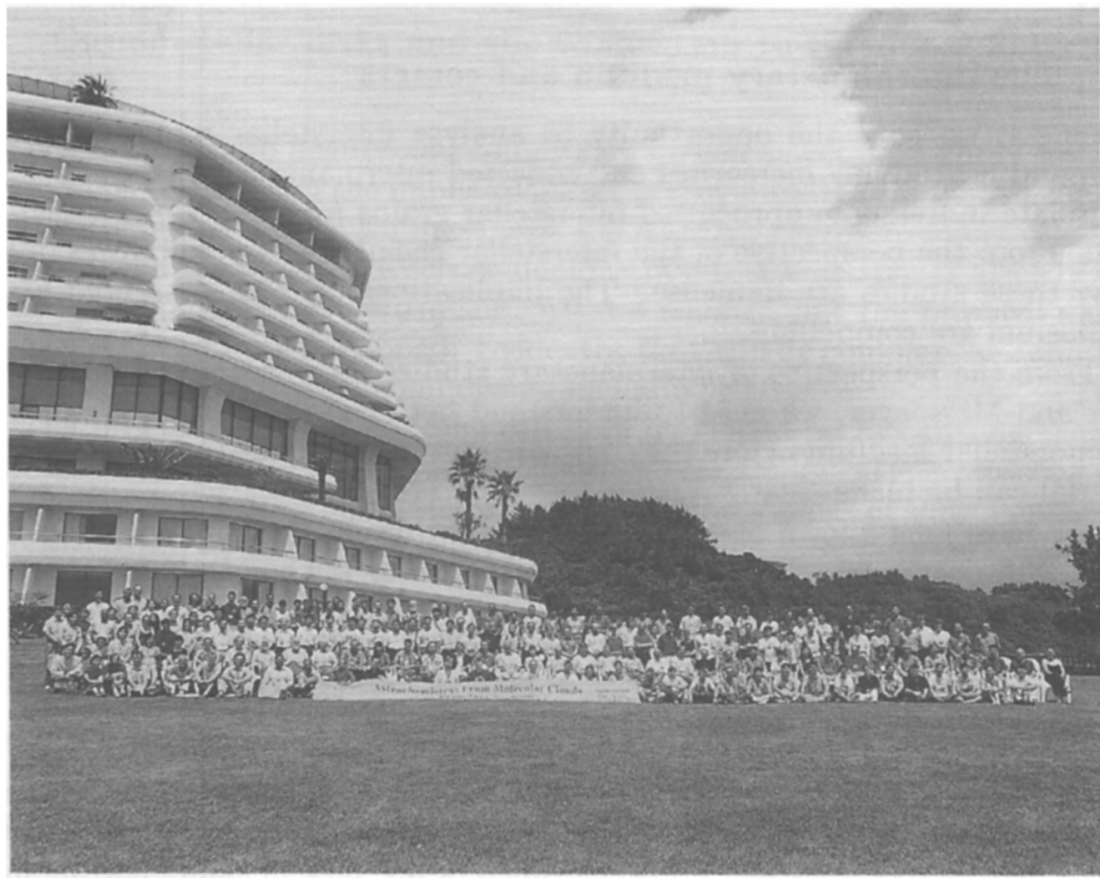

